\title{
The comparison between combination of pancreatic stent and nsaid with nsaid alone in preventing post ERCP pancreatitis
}

\begin{abstract}
ERCP as a diagnostic and therapeutic tool for biliary tract and pancreatic diseases is associated with many complications such as Pancreatitis, bleeding, perforation, and cardiovascular consequences. A recent survey showed that the use of non-steroid antiinflammatory drugs before ERCP could reduce risk of pancreatitis after ERCP. In order to assess this hypothesis in the patients admitted to Taleghani hospital, Tehran, Iran, with CBD stone, we designed the current study. After randomized selection and division of the patients to different groups; the patient's candidate for pancreatic duct stent (PD stent) and Indomethacin-supplement procedure and the patients under Indomethacin-supplement process alone. After evaluating different variabilities as sex, age, Post ERCP Pancreatitis (PEP) with use of PD stent, PEP with use of indomethacin-supplement and PD stent, medications, type of disease, laboratory test such as CBC, AST, ALT and bilirubin test, ERCP was conducted by one expert specialist. Blinding of the staff and analysis department was conducted as well. Data analysis showed that $<10$ minutes of cannulation could decrease the incidence of PEP in our study ( $\mathrm{P}$-value $<0.05$ ), while other variables did not manifested any correlation with the incidence of PEP. In conclusion, we showed the evidence that different interventional elements or patient associated risk factors had no significantly effect on PEP incidence. However, deep cannulation time as the important factor of procedure could alter the rate of PEP.
\end{abstract}

Keywords: post ercp pancreatitis, pancreatic duct stent, indemethacin
Special Issue - 2018

\author{
Alizadeh Arash Heidarnezhad, Amir \\ Houshang Mohammad \\ Department of Medical Science, University of Shahid Beheshti, \\ Iran \\ Correspondence: Mohammad Alizadeh AH, Department \\ of Medical Science, University of Shahid Beheshti, Taleghan \\ Hospital, Parvaneh Ave, Tabnak Str, Evin, Tehran, Iran- \\ 19857|74|3, Tel 0098-2I-2243252 I, Fax 0098-2I-224325 I7, \\ Email ahmaliver@yahoo.com
}

Received: May 26, 2017 | Published:September 19,2018
Abbreviations: PEP, post ercp pancreatitis; ERCP, endoscopic retrograde cholangio pancreatography

\section{Introduction}

Endoscopic retrograde cholangio pancreatography (ERCP) is one of the safest and most effective procedures that applied frequently in the management of pancreatobiliary disorders. ${ }^{1}$ Wide range of complications depending on the type of intervention used, still remain after ERCP. The most important complications of this procedure could be infectious, hemorrhage, perforation and post-ERCP pancreatitis (PEP). ${ }^{2,3}$ One of the most common complications is acute pancreatitis that imposes major problems such as morbidity, occasional death and increasing of health care expenditures to the patients. ${ }^{4,5}$

The occurrence of post-ERCP pancreatitis ranges in different studies depending on patient selection, reported from 1-15.7\%, however this amount at high-risk patients reaches to $30 \% .^{6-8}$ the mechanisms that lead to post-ERCP pancreatitis are complex and not fully understood. Rather than having a single pathogenesis, post-ERCP pancreatitis is believed to be multi-factorial, involving a combination of chemical, hydrostatic, enzymatic, mechanical, and thermal factors. Although there are some uncertainties in predicting which patients would develop acute pancreatitis following ERCP, a number of risk factors acting independently or in concert have been proposed as predictors of post-ERCP pancreatitis. ${ }^{9-12}$

Preventing pancreatitis after ERCP remains an important clinical issue. Progress in the last two decades has led to substantive reductions in PEP rates due to more appropriate patient selection, improved procedural techniques, and the adoption of prophylactic pancreatic duct (PD) stent in high-risk cases. Pancreatic duct stones are found in 22 to 60percent of patients with chronic pancreatitis. The stones can lead to obstruction of outflow of pancreatic secretions, causing increased intraductal pressure. Because the pancreas is relatively noncompliant, the rise in intraductal pressure can induce tissue hypertension and ischemia, which may be a major factor causing pain in patients with chronic pancreatitis. ${ }^{13-18}$

PD stent placement, introduced in the late $1990 \mathrm{~s}$, has become a common clinical practice in the United States and is widely regarded as an effective means of preventing PEP in high-risk cases. While PD stent placement clearly reduces risk, it remains technically challenging, time consuming, and costly. Moreover, attempting to place a PD stent with subsequent failure actually increases the risk of PEP above baseline by inducing injury to the pancreatic orifice. ${ }^{19-24}$

Arecent survey showed that the use of non-steroid anti-inflammatory drugs before ERCP could reduce the risk of pancreatitis after ERCP. The physiopathology of Indomethacin induced pancreatitis has not yet been elucidated but, as suggested by Memis it probably implicates a decrease in glutathione levels, a decrease in superoxide activities and increased oxidative stress. ${ }^{25}$ Different studies were performed to find solutions for prevention of PEP, however no method and medication for reducing PEP effects has been definitively proven yet. Wireguided biliary cannulation, placement of a pancreatic duct stent and pharmacotherapy are the most important preventive methods which have been discussed. Conflicting reports about the use of each of these methods is reported. It was confirmed that placing stent in pancreatic tracts can play a decisive role in reducing the severity of pancreatitis after ERCP in high risk patients. ${ }^{26,27}$ 
Several studies have demonstrated that NSAIDs can reduce complications of ERCP. In a report in Iran determine that PEP has no preventive effect for allopurinol. Dr SotoudeManesh showed that beneficial effect of $100 \mathrm{mg}$ Indomethacin in prevention of PEP. Cheon $\mathrm{Y}$ have shown that younger age $(<65$ years $)$ and female sex were significantly associated with an increased risk of PEP. There is no study at this subject in Iran. So in this study we tried to consider the prophylactic role and evaluation of pancreatic duct stent compared with rectal Indomethacin administration in reducing PEP.

\section{Material and methods}

\section{Patient screening and selection}

Every patient with CBD stone who refer to Taleghani hospital for ERCP was considered to the current study. All patients were divided into two groups applying a randomized method: The patients under PD stent and Indomethacin-supplement procedure in versus the patients under Indomethacin-supplement process. Clinical and demographic characterized of every patients was registered in a questioner. Laboratory tests including: biochemical test (ALT, AST, bilirubin), immunological test such as C-reactive protein (CRP), total blood count $(\mathrm{CBC})$ was performed before the procedure on selected patients. Also, the serum amylase and lipase level was measured $24 \mathrm{~h}$ after performing ERCP again.

\section{ERCP procedure and follow up}

Post ERCP pancreatitis (PEP) was defined as persistent abdominal pain in accordance with pancreatitis along with serum amylase or lipase increased 3-fold ( $\geq 3$ ), 24hours after ERCP.

\section{Blinding evaluation of the patients}

Analysis department staffs were blinded due to their unawareness of the patient's subgroup.

\section{Statistical analysis}

Statistical analysis was conducted by SPSS (version 21, Co Ltd, Tokyo, Japan). Differences between groups were considered to be significant if $\mathrm{p}$-values were less than 0.05. Significant association was estimated using chi square or fisher exact tests in pre and post ERCP procedure results.

\section{Results}

\section{Evaluation of probable patient-related risk factors}

After the administration of the selected patients to the study their related risk factors and probable relationship with the incidence of PEP was considered. As shown in Table 1, age, sex, smoking, alcoholism, addiction to opium, abdominal pain, past cholecystectomy, history of PEP, diabetes and chronic pancreatitis were taken by the history patients and their family provided by documents. As mentioned, the patients were subdivided into the groups using a randomized excel method. Then, their risk factors were compared between the groups manifesting no significant relation between any of the variabilities and the risk of PEP.

Table I Of patient-related risk factors in subgroups and relationship them with incidence of PEP.The frequency

\begin{tabular}{|c|c|c|c|c|c|c|}
\hline Variables & Biliary stent & PD stent & Indomethacin+PD Stent & Total of patients & Incidence of PEP & $P$ value \\
\hline Female/male & $29 / 31$ & $53 / 47$ & $92 / 108$ & $92 / 108$ & $13 / 17$ & 0.241 \\
\hline Smoker(yes/no) & $19 / 4 \mid$ & $28 / 74$ & $58 / 142$ & $86 / 114$ & 2I-Sep & 0.522 \\
\hline Age(<60years/>60years) & $19 / 11$ & $48 / 52$ & $98 / 102$ & $98 / 102$ & $19 / 11$ & 0.437 \\
\hline Alcoholism (yes/no) & Mar-57 & May-95 & $8 / 192$ & $8 / 192$ & 27-Mar & 0.263 \\
\hline Opium addict(yes/no) & $21 / 29$ & $24 / 76$ & $38 / 162$ & $38 / 162$ & $14 / 16$ & 0.546 \\
\hline Abdominal pain(yes/no) & $36 / 34$ & $65 / 35$ & $92 / 108$ & $92 / 108$ & $26 / 4$ & 0.072 \\
\hline Cholecystectomy (yes/no) & $26 / 34$ & $25 / 75$ & $46 / 154$ & $46 / 154$ & $15 / 15$ & 0.758 \\
\hline History of PEP(yes/no) & Jun-44 & $8 / 192$ & $19 / 18 \mid$ & $19 / 18 \mid$ & 22-Aug & 0.638 \\
\hline DM(yes/no) & Dec-38 & $26 / 74$ & $38 / 162$ & $38 / 162$ & 2I-Sep & 0.462 \\
\hline Chronic-pancreatitis(yes/no) & Jun-54 & $7 / 193$ & $2 / 198$ & $2 / 198$ & 27-Mar & 0.568 \\
\hline Cardiopulmonary (yes/no) & Nov-49 & Jun-94 & $17 / 183$ & $17 / 183$ & 27-Mar & 0.865 \\
\hline
\end{tabular}

*=significant association

Note The number of patients in each group is as follows, biliary stent $(n=60)$, PD stent + indomethacin $(n=100)$, total of patients $(n=200)$ and incidence of PEP $(n=30)$

Laboratory findings of subgroups before ERCP procedure

In order to understand the probable differences between the laboratory tests of the patients before any ERCP procedure, all the patients were evaluated by their blood sample. The staff taking the blood samples was unaware of the patient's subgroup. Samples were analyzed by the laboratory department of the hospital for AST, ALT, the ratio AST/ALT, HB, WBC, CRP and PALT, showing no significant difference between the lab data of PEP incidences and non-PEP patients group (Table 2).

\section{Consideration of applied interventional variables and PEP occurrence rate}

Due to the possible effect of different interventional methods or time of procedure, we asses applied procedural methods as biliary stent used patients, PD with/without indomethacin administration groups, PD guide wire, the time of deep cannulation, balloon dilation of S.O. 
and biliary sphincterotomy incidence relation with PEP incidence (Table 3). Data analysis revealed $<10$ minutes of deep cannulation significant impact on reducing the incidence of PEP ( $p$-value $<0.05$ ). The other interventional factors did not proved any significant relation due to the occurrence of PEP. These data may suggest that being an expert and fast operating specialist as the conductor of the procedure could decrease the incidence of PEP, while other elements of the interventions did not alter the incidence.

Table 2 The mean comparison of pre ERCP laboratory findings variables between subgroups (mean \pm S.E.M)

\begin{tabular}{llll}
\hline Variables & PEP & Without PEP & P-value \\
\hline ALT & $117.82 \pm 19$ & $86 \pm 5.3$ & 0.195 \\
AST & $98.3 \pm 16.8$ & $73.9 \pm 4.9$ & 0.087 \\
AST /ALT & $1.25 \pm 1.1$ & $1.2 \pm 0.11$ & 0.64 \\
HB & $13.3 \pm 0.45$ & $12.6 \pm 0.95$ & 0.86 \\
WBC & $10.05 \pm 1.27$ & $8.9 \pm 0.91$ & 0.56 \\
CRP & $26.7 \pm 8.5$ & $17.4 \pm 3.5$ & 0.91 \\
PLAT & $245.08 \pm 25.5$ & $235.06 \pm 7.5$ & 0.83
\end{tabular}

*=significant association

Note Incidence of PEP $(n=30)$, individuals without PEP $(n=170)$

Regarding ERCP findings of the groups and PEP incidence

Different findings of the ERCP technique were gathered and Table 3 The relationship between applied interventional variables and incidence of PEP in patients analyzed from different patient groups, comparing with the significant alter in PEP incidence. As shown in Table 4, CBD diameter dilatation, stricture/stone of CBD, PD dilatation or its irregularity, malignancy, CBD cyst, diverticulum were appraised showing no significant correlation with PEP incidence between different groups of the study (Biliary stent, PD stent, Indomethacin +PD stent).

\section{Laboratory assessment of the patients before ERCP}

As demonstrated in Figure 1, laboratory tests of the patients with PEP and without PEP complication did not show any significant relation with any of the tests (AST, ALT, CRP, WBC, HB, and PALT). This may reveal its ineffective role in pre-operating ERCP and PEP incidence.

Figure I Diagram of mean comparison of pre-ERCP laboratory finding

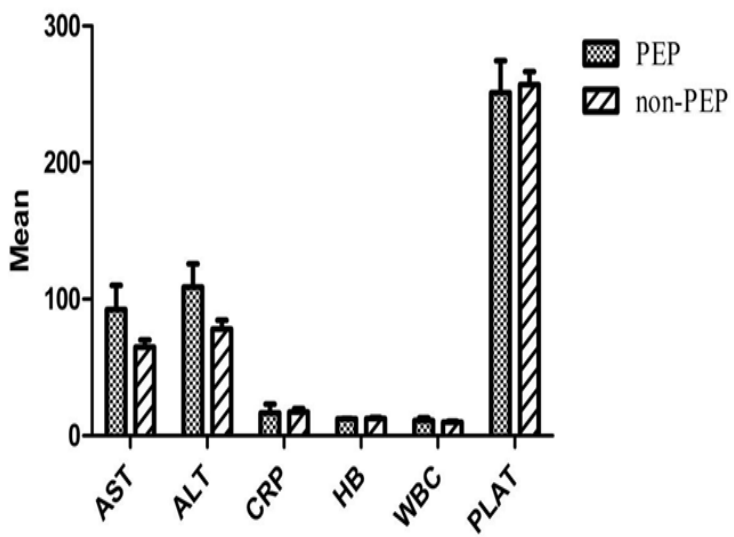

variables between patients with PEP complication and without it. There were no significance mean differences between serum lab findings including: AST (Aspartate transaminase), ALT (Alanine transaminase), CRP(C- reactive protein), HB (Hemoglobin), WBC (white blood cells) and PLAT (Platelet) between patients with PEP complication and without it.

\begin{tabular}{|c|c|c|c|c|}
\hline Variables & Incidence of pep & P-value & OR & $95 \% \mathrm{Cl}$ \\
\hline \multicolumn{5}{|l|}{ Placement of stent } \\
\hline Biliary stent & $13(43.3 \%)$ & 0.93 & 0.964 & $0.39-2.3$ \\
\hline PD stent + Rectal Indomethacin & II(36.6\%) & 0.55 & 0.74 & $0.281-1.9$ \\
\hline Administration of rectal & $19(63.3 \%)$ & 0.55 & 1.3 & $0.5-3.5$ \\
\hline \multicolumn{5}{|l|}{ Indomethacin } \\
\hline PD Guide Wire & $18(60.0 \%)$ & 0.2 & 1.7 & $0.74-3.9$ \\
\hline \multicolumn{3}{|l|}{ Time of deep cannulation } & - & - \\
\hline$<10$ min & $4(13.3 \%)$ & $0.005^{*}$ & 3.41 & $0.9-4.2$ \\
\hline $10-30 \mathrm{~min}$ & $9(30.0 \%)$ & 0.63 & 1.3 & $0.6-3.6$ \\
\hline$>30 \mathrm{~min}$ & $17(56.6 \%)$ & 0.33 & 1.1 & $0.5-3.3$ \\
\hline Balloon dilation of S.O & $9(30.0 \%)$ & 0.6 & 1.2 & $0.47-3.1$ \\
\hline Biliary sphincterotomy & $16(53.3 \%)$ & 0.82 & 0.9 & $0.39-2.1$ \\
\hline
\end{tabular}

*=significant association

Note Incidence of PEP $(n=30)$ 
Table 4 The frequency of ERCP finding variables in subgroups and correlations these findings with incidence of PEP

\begin{tabular}{|c|c|c|c|c|c|c|}
\hline Variables & Biliary stent & PD Stent & Indomethacin +PD & Total of I patients & Incidence of PEP & p-value \\
\hline GB dilation & $2(3.3 \%)$ & $3(3.0 \%)$ & $7(3.5 \%)$ & $7(3.5 \%)$ & $4(13.3 \%)$ & 0.77 \\
\hline CBD dilatation & $43(71.6 \%)$ & $73(73 \%)$ & $76(76 \%)$ & II4(57.0\%) & $19(63.3 \%)$ & 0.132 \\
\hline \multicolumn{7}{|c|}{ Diameter of CBD dilatation } \\
\hline$<10 \mathrm{~mm}$ & $36(60.0 \%)$ & $3 \mathrm{I}(3 \mathrm{I} .0 \%)$ & $58(58.0 \%)$ & $89(44.5 \%)$ & $26(86.6 \%)$ & 0.84 \\
\hline$>10 \mathrm{~mm}$ & $32(53.3 \%)$ & $56(56.0 \%)$ & $61(61.0 \%)$ & $117(58.5)$ & $8(26.6 \%)$ & \\
\hline $\mathrm{CBD}$ stricture & $19(31.6 \%)$ & $22(22 \%)$ & $29(29 \%)$ & $5 \mathrm{I}(25.5 \%)$ & $16(53.3 \%)$ & 0.07 \\
\hline CBD stone & $32(53.3 \%)$ & $48(48.0 \%)$ & $58(58.0 \%)$ & $106(53.0 \%)$ & $17(56.6 \%)$ & 0.35 \\
\hline PD dilatation & $10(16.6 \%)$ & $\mathrm{II}(\mathrm{II} .0 \%)$ & $9(9.0 \%)$ & $20(10.0 \%)$ & $2(6.6 \%)$ & 0.62 \\
\hline PD irregularity & $3(5 \%)$ & $7(7 \%)$ & $6(6 \%)$ & $13(6.5 \%)$ & $2(6.6 \%)$ & 0.93 \\
\hline Malignancy & $10(16.6 \%)$ & $22(22.0 \%)$ & $25(25.0 \%)$ & $47(23.9 \%)$ & $9(30.0 \%)$ & 0.36 \\
\hline $\mathrm{CCA}$ & $20(12.0 \%)$ & $4(4.0 \%)$ & $16(16 \%)$ & $20(10 \%)$ & $26(87.0 \%)$ & 0.69 \\
\hline Ampulory & $2(3.3 \%)$ & $3(3.0 \%)$ & $4(4.0 \%)$ & $7(3.5 \%)$ & $9(30 \%)$ & 0.4 \\
\hline Diverticulum & $4(6.6 \%)$ & $2(2.0 \%)$ & $7(7 \%)$ & $9(4.5 \%)$ & $5(16.6 \%)$ & 0.81 \\
\hline CBD cyst & $2(3.3 \%)$ & $3(3.0 \%)$ & $\mathrm{I}(\mathrm{I} .0 \%)$ & $4(2.0 \%)$ & 0 & 0.55 \\
\hline
\end{tabular}

*=significant association

Note The number of patients in each group is as follows, biliary stent $(n=60), P D$ stent

+indomethacin $(n=100)$, total of patients $(n=200)$ and incidence of $\operatorname{PEP}(n=30)$

\section{Discussion}

As the most common complication of ERCP, PEP accounts for a huge financial burden to the hospitals and can cause the deterioration of patient's health. ${ }^{28}$ Here, we investigated short-term prophylactic PD stent and rectal indomethacin to prevent PEP in patients with different biliary disorders. Our data suggested that the use of prophylactic rectal indomethacin in combination with PD stents has decreased the rate of PEP in the selected patients, however, the outcome was not significantly different from using rectal indomethacin alone.

In relation to patient-related risk factors, previous studies have discussed that being female and younger ages were significant risk factors for PEP. ${ }^{29}$ The impact of age seems to be related to protective effect of progressive decline in pancreatic exocrine function by aging. ${ }^{30}$ In addition to PEP, overall complications of ERCP were 1.52times higher in women. ${ }^{29}$ In contrast, there was no significant association between age or sex and PEP in our study, even slightly higher risk of PEP, but not significant, was observed. Interestingly, lack of association between age and sex and the risk of PEP also has been observed by Cheng et al. ${ }^{31}$ In the searching for patient-related risk factors, previous evidences has reported that post-ERCP elevation of inflammatory and biochemical markers could be a potential predictors of PEP. ${ }^{32}$ It has been also reported that pre procedure ESR $>30 \mathrm{~mm} /$ hour is a significant factor that can predict increased risk of PEP. ${ }^{33}$ Our evaluation of pre-ERCP level of biochemical markers indicated that there was no significant relationship between these markers prior to ERCP and the risk of elevated PEP.

Procedure-related risk factors and subsequent prophylactic strategies are the other side of post-ERCP pancreatitis prevention. Earlier studies have reported biliary sphincterotomy and balloon dilation of S.O. are the risk factors for developing post-ERCP pancreatitis. ${ }^{34}$ However, later reports have not mentioned these variables as significant risk factors of PEP. ${ }^{29}$ Although they alternatively added other risk factors, such as cannulation time and periampullary diverticulum, as significantly related to pancreatitis. ${ }^{29}$ Our observation suggested that balloon dilation of S.O. and biliary sphincterotomy were not related to higher risk of PEP. Interestingly, our data indicated a direct and significant relationship between increasing of the time of deep cannulation with increased risk of pancreatitis. The best rate of PEP was related to the procedures in which deep cannulation time was under 10 minutes and this was independent of other patient or procedure-related risk factors.

Nonsteroidal anti-inflammatory drugs (NSAIDs) are potent inhibitors of phospholipase $\mathrm{A}^{2}$, cyclooxygenase, and neutrophilendothelial interactions, all believed to play an important role in the pathogenesis of acute pancreatitis. ${ }^{35,36}$ A large body of evidence has recommended NSAIDs for preventing PEP, predominantly in high risk cases. ${ }^{37}$ On the other hand, PD stent has been introduced as another safe and effective option to prevent PEP if the operation indications are well controlled. ${ }^{38}$ Combination prophylaxis by NSAIDs and PD stents has been investigated and the results suggested superior effects of NSAID prophylaxis alone or insignificant difference in comparison to combination prophylaxis. ${ }^{39,40}$ Consistently, our data indicated no significant reduction of PEP by combination of rectal indomethacin prophylaxis with PD stent.

\section{Conclusion}

Overall, here we represent the evidence that the most of procedural or patient associated risk factors in developing PEP had not significantly affected the outcome. Still, deep cannulation time as the important part of procedure has the capacity to alter the rate of PEP by a wide range. PD stent as a prophylactic operation should be considered in parallel by other cost beneficent prophylaxis such as pre-ERCP administration of rectal indomethacin. 


\section{Acknowledgements}

None.

\section{Conflict of interest}

There is no conflict of interest in any fields of the study.

\section{References}

1. Freeman ML, Guda NM. Prevention of post-ERCP pancreatitis: a comprehensive review. Gastrointest Endosc. 2004;59(7):845-864.

2. Mohammad Alizadeh AH, Afzali ES, Behzad C, et al. Is ESR Important for Predicting Post-ERCP Pancreatitis?. Clin Med Insights Gastroenterol. 2015;8:23-27.

3. Nicholas M Szary, Firas H Al-Kawas. Complications of endoscopic retrograde cholangio pancreatography: How to avoid and manage them Gastroenterol Hepatol (NY). 2013;9(8):496-504.

4. Freeman ML, Nelson DB, Sherman S, et al. Complications of endoscopic biliary sphincterotomy. $N$ Engl J Med. 1996;335(13):909-918.

5. Choudhary A, Bechtold ML, Arif M, et al. Pancreatic stents for prophylaxis against post-ERCP pancreatitis: a meta-analysis and systematic review. Gastrointest Endosc. 2011;73(2):275-282.

6. Barthet M, Lesavre N, Desjeux A, et al. Complications of endoscopic sphincterotomy: results from a single tertiary referral center. Endoscopy. 2002;34(12):991-997.

7. Cotton PB, Garrow DA, Gallagher J, et al. Risk factors for complications after ERCP: a multivariate analysis of 11,497 procedures over 12 years. Gastrointest Endosc. 2009;70(1):80-88.

8. Elmunzer BJ, Scheiman JM, Lehman GA, et al. A randomized trial of rectal indomethacin to prevent post-ERCP pancreatitis. $N$ Engl $J$ Med 2012;366(15):1414-1422.

9. Freeman ML, DiSario JA, Nelson DB, et al. Risk factors for post-ERCP pancreatitis: A prospective, multicenter study. Gastrointest Endosc. 2001;54(4):425-434.

10. Chi Liang Cheng, Stuart Sherman, James L Watkins, et al. Risk factors for post-ERCP pancreatitis: a prospective multicenter study. The American Journal of Gastroenterology. 2006;101:139-147.

11. Mehta SN, Pavone E, Barkun JS, et al. Predictors of post-ERCP complications in patients with suspected choledocholithiasis. Endoscopy. 1998;30(5):457-463.

12. Naitoh I, Ohara H, Nakazawa T, et al. Unilateral versus bilateral endoscopic metal stenting for malignant hilar biliary obstruction. J Gastroenterol Hepatol. 2009;24(4):552-557.

13. Petrov MS, Savides TJ. Systematic review of endoscopic ultrasonography versus endoscopic retrograde cholangiopancreatography for suspected choledocholithiasis. Br J Surg. 2009;96(9):967-974.

14. Romagnuolo J, Bardou M, Rahme E, et al. Magnetic resonance cholangiopancreatography: a meta-analysis of test performance in suspected biliary disease. Ann Intern Med. 2003;139(7):547-557.

15. Sherman S, Troiano FP, Hawes RH, et al. Sphincter of Oddi manometry: Decreased risk of clinical pancreatitis with use of a modified aspirating catheter. Gastrointest Endosc. 1990;36(5):462-466.

16. Cennamo V, Fuccio L, Zagari RM, et al. Can a wire-guided cannulation technique increase bile duct cannulation rate and prevent post-ERCP pancreatitis: A meta-analysis of randomized controlled trials. Am J Gastroenterol. 2009;104(9):2343-2350.
17. Gregory A. Cote, Michael Ansstas, Rishi Pawa, et al. Difficult biliary cannulation: use of physician controlled wire-guided cannulation over a pancreatic duct stent to reduce the rate of precut sphincterotomy (with video). Gastrointest Endosc. 2010;71(2):275-279.

18. Freeman ML. Pancreatic stents for prevention of post-endoscopic retrograde cholangiopancreatography pancreatitis. Clin Gastroenterol Hepatol 2007;5(11):1354-1165.

19. Brackbill S, Young S, Schoenfeld P, et al. A survey of physician practices on prophylactic pancreatic stents. Gastrointest Endosc. 2006;64(1):45-52.

20. Fazel A, Quadri A, Catalano MF, et al. Does a pancreatic duct stent prevent post-ERCP pancreatitis? A prospective randomized study. Gastrointest Endosc. 2003;57(3):291-294.

21. Zolotarevsky E, Fehmi SM, Anderson MA, et al. Prophylactic 5-Fr pancreatic duct stents are superior to 3-Fr stents: a randomized controlled trial. Endoscopy. 2011;43(4):325-330.

22. Freeman ML, Overby C, Qi D. Pancreatic stent insertion: consequences of failure and results of a modified technique to maximize success. Gastrointest Endosc. 2004;59(1):8-14.

23. Mahjoub W, Jarboui S, Ben Moussa M, et al. Indomethacin-induced pancreatitis; a second case report. JOP. 2006;7(3):321-323.

24. Drake DH, Fry WJ. Ductal drainage for chronic pancreatitis. Surgery. 1989;105(2):131-140

25. Bradley EL. Pancreatic duct pressure in chronic pancreatitis. Am J Surg. 1982;144(3):313-336

26. Goran Hauser, Marko Milosevic, Davor Stimac, et al. Preventing post-endoscopic retrograde cholangiopancreatography pancreatitis: What can be done? World J Gastroenterology. 2015;21(4):1069-1080.

27. Wang P, Li ZS, Liu F, et al. Risk factors for ERCP-related complications: a prospective multicenter study. Am $J$ Gastroenterology. 2009;104(1):31-40.

28. Laugier R, Bernard JP, Berthezene $P$, et al. Changes in pancreatic exocrine secretion with age: pancreatic exocrine secretion does decrease in the elderly. Digestion. 1991;50(3-4):202-211.

29. Bulent Güngör, Kaslm Çağlaya, Cafer Polat, et al. The predictivity of serum biochemical markers in acute biliary pancreatitis. ISRN gastroenterology. 2011;2011:279607.

30. Messmann H, Vogt W, Holstege A, et al. Post-ERP pancreatitis as a model for cytokine induced acute phase response in acute pancreatitis. Gut. 1997;40(1):80-85.

31. Freeman ML, DiSario JA, Nelson DB, et al. Risk factors for post-ERCP pancreatitis: a prospective, multicenter study. Gastrointestinal endoscopy. 2001;54(4):425-434.

32. Gross V, Leser HG, Heinisch A, et al. Inflammatory mediators and cytokines--new aspects of the pathophysiology and assessment of severity of acute pancreatitis?. Hepatogastroenterology. 1993;40(6):522-530.

33. Mäkelä A, Kuusi T, Schröder T. Inhibition of serum phospholipase-A2 in acute pancreatitis by pharmacological agents in vitro. Scandinavian journal of clinical and laboratory investigation. 1997;57(5):401-407.

34. Puig I, Calvet X, Baylina M, et al. How and when should NSAIDs be used for preventing post-ERCP pancreatitis? A systematic review and meta-analysis. PloS one. 2014;9(3):e92922.

35. Fan JH, Qian JB, Wang YM, et al. Updated meta-analysis of pancreatic stent placement in preventing post-endoscopic retrograde cholangiopancreatography pancreatitis. World $J$ of Gastroenterology. 2015;21(24):7577-7583. 
36. Elmunzer BJ, Higgins PD, Saini SD, et al. Does rectal indomethacin eliminate the need for prophylactic pancreatic stent placement in patients undergoing high-risk ERCP\&quest; Post hoc efficacy and cost-benefit analyses using prospective clinical trial data. Am $J$ Gastroenterology. 2013;108(3):410-415.

37. Akbar A, Dayyeh BK, Baron TH, et al. Rectal nonsteroidal anti-inflammatory drugs are superior to pancreatic duct stents in preventing pancreatitis after endoscopic retrograde cholangiopancreatography: a network meta-analysis. Clin Gastroenterology and Hepatology. 2013;11(7):778-783.
38. Freeman ML, Guda NM. Prevention of Post-ERCP Pancreatitis: a comprehensive review. Gastrointest Endosc. 2004;59:845-64

39. Choudhary A, Bechtold ML, Arif M, et al. Pancreatic stents for prophylaxis against post-ERCP pancreatitis: a meta-analysis and systematic review. Gastrointest Endosc. 2011;73:275-82.

40. Cheng CL, Sherman S, Watkins JL, et al. Silverman WB. Risk factors for post-ERCP pancreatitis: a prospective multicenter study. The American journal of gastroenterology. 2006;101(1):139-47. 